\title{
Observation of Supersymmetry in Rigid Symmetric Top Rotor
}

\author{
Hosung Sun \\ Department of Chemistry, Sungkyunkwan University, Suwon 440-746, Korea.E-mail: hsun@skku.edu \\ Received January 6, 2006
}

\begin{abstract}
It is explicitly shown that a supersymmetry structure exists in the spectrum of a rigid symmetric top rotor in the molecule-fixed frame. Using projection operators constructed from the time-reversal symmetry of the rotor, the full rotor Hamiltonian is separated into two parts, i.e., the bosonic and fermionic components. The construction, without ambiguity, suggests that the rotor has a supersymmetry in it. This supersymmetry is mathematically equivalent to that of the free rotor on a plane recently noted by Rau.
\end{abstract}

Key Words : Supersymmetry, Symmetric top, Rotation

\section{Introduction}

Though solid and fundamental experimental evidences of supersymmetry in nature have yet to come, ${ }^{1,2}$ there have been many efforts to find the supersymmetry in real physical systems. Due to the complexity of real systems and/or the spontaneous breaking of supersymmetry, the exact supersymmetry may not be found but there are several examples reported. ${ }^{3}$ These examples are approximate but are not trivial at all. Rather they cast a profound implication in understanding the nature. For example, an approximate supersymmetry is observed at the spectra of complex nuclei (Os-Ir, etc. $)^{4,5}$ And singularities in a randomly diluted ferromagnet are explained. ${ }^{6,7}$ Notably, a supersymmetry is found in the electronic energy spectra of hydrogen atom and its isoelectronic ions, and a similar behavior is also noted in alkali atoms. ${ }^{8-13}$ The hydrogen atom under external field has also a supersymmetric structure and the relativistic hydrogen atom does too. ${ }^{14-17}$ Many examples are compiled in References 18 and 19 .

As another example of supersymmetry, we suggest that the rotation of a rigid symmetric top molecule must have a supersymmetric spectrum. As well known, ${ }^{20-23}$ the moleculefixed angular momentum of a symmetric top rotor is quantized along the figure axis. This quantization produces a double degeneracy of eigenstates apart from the degeneracy by quantization along the space-fixed principal axis. The double degeneracy is due to the time-reversal symmetry. That is, a molecule rotates one way or the other and the two rotations are identical when the molecule is symmetric top. Surely it implies that there could be a supersymmetry in this system. The simpler but identical to the symmetric top rotor system is the rotation of a body on a two-dimensional plane that Rau noted very recently. ${ }^{24}$

The requirements for a Hamiltonian $H$ being supersymmetric are as follows: ${ }^{3}$ (i) There is a zero-energy nondegenerate ground eigenstate of $H$. (ii) All the excited states are doubly degenerate, i.e., the states are in pairs. (iii) There should exist supersymmetry generating operators (or supercharges) $Q$ and $Q^{+}$that transform the degenerate pairs into one another and yield zero upon acting on the ground eigenstate. (iv) The $Q$ and $Q^{+}$should satisfy the following closed superalgebra, i.e., $[H, Q]=\left[H, Q^{+}\right]=0, H=\left\{Q^{+}, Q\right\}$, and $\{Q, Q\}=\left\{Q^{+}, Q^{+}\right\}=0$. The commutator [,] and the anticommutator $\{$,$\} are defined as \left[O_{1}, O_{2}\right]=O_{1} O_{2}-O_{2} O_{1}$ and $\left\{O_{1}, O_{2}\right\}=O_{1} O_{2}+O_{2} O_{1}$. In this work we explicitly show that the symmetric top rotor system fulfills the above supersymmetry requirements.

\section{Symmetric top rotor}

The Hamiltonian for rotational motion of a rigid body (or a molecule) can be expressed $\mathrm{as}^{21}$

$$
H=\frac{\hat{J}_{x}^{2}}{2 \mathrm{I}_{x}}+\frac{\hat{J}_{y}^{2}}{2 \mathrm{I}_{y}}+\frac{\hat{J}_{z}^{2}}{2 \mathrm{I}_{z}}=\mathrm{B} \hat{J}_{x}^{2}+\mathrm{C} \hat{J}_{y}^{2}+\mathrm{A} \hat{J}_{z}^{2}
$$

The three principal moments of inertia, $\mathrm{I}_{a}, \mathrm{I}_{b}, \mathrm{I}_{c}$, which lie on principal axes fixed in the molecule, completely determine the rotational properties. The exact relation between $a, b, c$ and $x, y, z$, depends on the system but can be left free for convenience. The $x, y, z$ are the coordinates in the moleculefixed (or the body-fixed) frame.

When the rotational constants $\mathrm{B}=\mathrm{C} \neq \mathrm{A}$, the molecule is called a symmetric top rotor. Let the $z$-axis be the figure axis whose principal moment of inertia is different from the other two, then the Hamiltonian is

$$
H=\mathrm{B} \hat{J}_{x}^{2}+\mathrm{B} \hat{J}_{y}^{2}+\mathrm{A} \hat{J}_{z}^{2}=\mathrm{B} \hat{J}^{2}+(\mathrm{A}-\mathrm{B}) \hat{J}_{z}^{2}
$$

where $\hat{J}^{2}=\hat{J}_{x}^{2}+\hat{J}_{y}^{2}+\hat{J}_{z}^{2}=\hat{J}_{X}^{2}+\hat{J}_{Y}^{2}+\hat{J}_{Z}^{2}$. The $X, Y, Z$ are the coordinates in the space-fixed frame. The eigenfunction is represented symbolically as $|J K M\rangle$ where the quantum number $J$ is for $\hat{J}^{2}, M$ for $\hat{J}_{Z}$, and $K$ for $\hat{J}_{z}$. Since the quantum number $M$ represents the quantization of total angular momentum on the space-fixed $Z$-axis, it is out of concern in this work. Therefore the eigenfunction of interest can be simply written as $|J K\rangle$,

$$
|J K\rangle \equiv \psi_{J K}(\varphi)=N \exp (i K \varphi)
$$

where $\varphi$ is the rotation angle on the molecule-fixed $x y$-plane. $N$ contains all the other relevant quantities, i.e., the 
normalization constant and the functions depending on $J$ and M. Recall that $\hat{J}_{z}=-i \hbar \frac{\partial}{\partial \varphi}$.

Then the Schrödinger equation for the symmetric top rotor is

$$
\left[B \hat{J}^{2}+(\mathrm{A}-\mathrm{B}) \hat{J}_{z}^{2}\right] \psi_{J K}(\varphi)=E_{J K} \psi_{J K}(\varphi)
$$

where the rotational eigenenergy is

$$
E_{J K}=\left[\mathrm{B} J(J+1)+(\mathrm{A}-\mathrm{B}) K^{2}\right]
$$

with $J=0,1,2, \ldots$ and $K=0, \pm 1, \pm 2, \ldots, \pm J$. ( $\hbar=1$ is used throughout.) When $\mathrm{A}-\mathrm{B}>0$, the system is called the prolate symmetric top (e.g., $\left.\mathrm{CH}_{3} \mathrm{Cl}\right)$ and when $\mathrm{A}-\mathrm{B}<0$, it is the oblate symmetric top (e.g., $\mathrm{BF}_{3}$ ). From now on we consider only the prolate top case because all of the following arguments are equally applied to the oblate top. In Eq. (5), one sees that the rotational state (within a given $J$ ) has the zero energy non-degenerate ground state $(K=0)$ and the doubly degenerate excited states with $K$ and $-K$. The energy levels of $|J K\rangle$ states, for a given $J$, are presented in Fig. 1. The exact double degeneracy occurs only when the symmetric top molecule is rigid. Of course, the real molecules are not rigid. However, the nonadiabatic couplings that break the symmetric top symmetry are usually so small that the couplings can be neglected. For instance, see the Reference 25 for $\mathrm{CH}_{3} \mathrm{Cl}$.

The double degeneracy arises because the rotational motion of a symmetric top molecule about the figure $z$-axis has the time-reversal symmetry..$^{20,21}$ Let $\hat{T}$ be the timereversal (complex conjugate) operator that is antiunitary, i.e.,

$$
\hat{T} \psi_{J K}(\varphi)=\psi_{J-K}(\varphi)=\psi_{J K}(-\varphi) \text {. }
$$

Of course the time-reversal operator acts only on the $\hat{J}_{z}^{2}$ motion, i.e., the rotation of molecule on the molecule-fixed $x y$-plane. Also one can easily find the following properties of the time-reversal and angular momentum operators, i.e.,

$$
\hat{T}^{2}=\hat{1} \text { and }\left\{\hat{T}, \hat{J}_{z}^{2}\right\}=0 .
$$

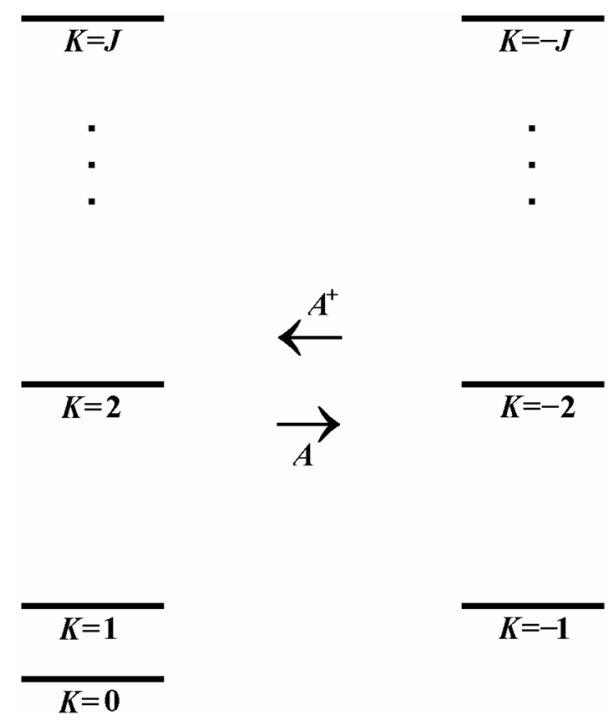

Figure 1. Rotational energy levels of a prolate symmetric top rotor, $|J K\rangle$ at a fixed $J$.
For a given $J$, the Hamiltonian $H$ may be expressed as a sum of two parts, i.e.,

$$
H=H_{S}+C_{J}, H_{S}=(\mathrm{A}-\mathrm{B}) \hat{J}_{z}^{2} \text { and } C_{J}=\mathrm{B} J(J+1) .
$$

The $H_{S}$ is the part that exhibits the time-reversal symmetry, while the constant term $C_{J}$ simply shifts the eigenenergy levels of $H_{S}$. When the $\hat{T}$ is acted upon the eigenstates of $H_{S}$, there are two subsets of states. One is a set of states $\left[\psi_{J K}^{(+)}(\varphi)\right]$ having the eigenvalue of +1 and the other $\left[\psi_{J K}^{(-)}(\varphi)\right]$ having the eigenvalue of -1 . In the following section we examine if the Hamiltonian $H_{S}$ satisfies the requirements for being supersymmetric.

\section{Supersymmetry}

The full eigenspace of $H_{S}$ can be separated into two subspaces of $H^{(+)}$and $H^{(-)}$. The $H^{(+)}$involves the $\psi_{J K}^{(+)}(\varphi)$ states and the $H^{(-)}$does the $\psi_{J K}^{(-)}(\varphi)$ states. The division can be accomplished by using the projection operators $\hat{\Pi}_{+}$and П- ,

$$
\hat{\Pi}_{+}=\frac{1}{2}(\hat{1}+\hat{T}) \text { and } \hat{\Pi}_{-}=\frac{1}{2}(\hat{1}-\hat{T}) .
$$

The projection operators have the following properties, i.e.,

$$
\begin{aligned}
& \hat{\Pi}_{+}^{2}=\hat{\Pi}_{+}, \hat{\Pi}_{-}^{2}=\hat{\Pi}_{-}, \hat{\Pi}_{+} \hat{\Pi}_{-}=\hat{\Pi}_{-} \hat{\Pi}_{+}=0, \text { and } \\
& \hat{\Pi}_{+}+\hat{\Pi}_{-}=\hat{1} .
\end{aligned}
$$

Also one immediately finds that

$$
\hat{\Pi}_{+} \hat{J}_{z}=\hat{J}_{z} \hat{\Pi}_{-} \text {and } \hat{\Pi}_{-} \hat{J}_{z}=\hat{J}_{z} \hat{\Pi}_{+} \text {. }
$$

The $\psi_{J K}^{(+)}(\varphi)\left(\psi_{J K}^{(-)}(\varphi)\right)$ should be the eigenfunctions of the Hamiltonian $H_{S}$ and the time-reversal related projection operator $\hat{\Pi}_{+}\left(\hat{\Pi}_{-}\right)$simultaneously so that

$$
\begin{aligned}
& \psi_{J K}^{(+)}(\varphi)=\frac{1}{\sqrt{2}}\left[\psi_{J K}(\varphi)+\psi_{J-K}(\varphi)\right] \text { and } \\
& \psi_{J K}^{(-)}(\varphi)=\frac{1}{\sqrt{2}}\left[\psi_{J K}(\varphi)-\psi_{J-K}(\varphi)\right] .
\end{aligned}
$$

Note that

$$
\begin{aligned}
& \hat{\Pi}_{+} \psi_{J K}^{(+)}(\varphi)=\psi_{J K}^{(+)}(\varphi), \hat{\Pi}_{-} \psi_{J K}^{(-)}(\varphi)=\psi_{J K}^{(-)}(\varphi), \text { and } \\
& \hat{\Pi}_{ \pm} \psi_{J K}^{(\mp)}(\varphi)=0 \text { and } \\
& \hat{J}_{z} \psi_{J K}^{(+)}(\varphi)=K \psi_{J K}^{(-)}(\varphi) \text { and } \hat{J}_{z} \psi_{J K}^{(-)}(\varphi)=K \psi_{J K}^{(+)}(\varphi) .
\end{aligned}
$$

The corresponding Schrödinger equations are

$$
\begin{aligned}
& H^{(+)} \psi_{J K}^{(+)}(\varphi)=(\mathrm{A}-\mathrm{B}) K^{2} \psi_{J K}^{(+)}(\varphi) \text { and } \\
& H^{(-)} \psi_{J K}^{(-)}(\varphi)=(\mathrm{A}-\mathrm{B}) K^{2} \psi_{J K}^{(-)}(\varphi) .
\end{aligned}
$$

The supersymmetry of $H_{S}$ can be explained as follows. ${ }^{26}$ 
We write $H_{S}$ in a matrix form of $H_{S}=\left(\begin{array}{cc}H^{(+)} & 0 \\ 0 & H^{(-)}\end{array}\right)$. The supercharges can be written as $Q^{+}=\left(\begin{array}{ll}0 & A^{+} \\ 0 & 0\end{array}\right)$ and $Q=\left(\begin{array}{ll}0 & 0 \\ A & 0\end{array}\right)$.

Then one immediately notices that $\{Q, Q\}=\left\{Q^{+}, Q^{+}\right\}=0$. If we set $H^{(+)}=A^{+} A$ and $H^{(-)}=A A^{+}$, the following two relations, i.e., $\left[H_{S}, Q\right]=\left[H_{S}, Q^{+}\right]=0 \& H_{S}=\left\{Q^{+}, Q\right\}$, are satisfied. (Requirement (iv) is fulfilled.)

The Hamiltonian $H_{S}$ is supersymmetric if we find appropriate expressions of the operators $Q$ (or $A$ ) and $Q^{+}$(or $A^{+}$). One of possible choices for $A$ and $A^{+}$is

$$
A=\sqrt{\mathrm{A}-\mathrm{B}} \hat{J}_{z} \hat{\Pi}_{+} \text {and } A^{+}=\sqrt{\mathrm{A}-\mathrm{B}} \hat{J}_{z} \hat{\Pi}_{-} .
$$

Let us verify if the choice is appropriate. The Hamiltonians $H^{(+)}$in (+)-subspace and $H^{(-)}$in (-)-subspace are, using Eqs. (10) and (11),

$$
\begin{aligned}
H^{(+)} & =A^{+} A=\left[\sqrt{\mathrm{A}-\mathrm{B}} \hat{J}_{z} \hat{\Pi}_{-}\right]\left[\sqrt{\mathrm{A}-\mathrm{B}} \hat{J}_{z} \hat{\Pi}_{+}\right] \\
& =(\mathrm{A}-\mathrm{B}) \hat{J}_{z}\left(\hat{\Pi}_{-} \hat{J}_{z}\right) \hat{\Pi}_{+}=(\mathrm{A}-\mathrm{B}) \hat{J}_{z}\left(\hat{J}_{z} \hat{\Pi}_{+}\right) \hat{\Pi}_{+} \\
& =(\mathrm{A}-\mathrm{B}) \hat{J}_{z}^{2} \hat{\Pi}_{+}
\end{aligned}
$$

and similarly,

$$
H^{(-)}=A A^{+}=(\mathrm{A}-\mathrm{B}) \hat{J}_{z}^{2} \hat{\Pi}_{-} .
$$

They are identical with the original $H_{S}$ but the projection operator plays a role of distinguishing the Hamiltonian $H^{(+)}$ from the partner Hamiltonian $H^{(-)}$.

The non-degenerate ground state $(K=0)$ has zero energy and it belongs to the (+)-subspace because of $\hat{\Pi}_{+} \psi_{J 0}^{(+)}(\varphi)=$ $\psi_{J 0}^{(+)}(\varphi)$ or $\psi_{J 0}^{(-)}(\varphi)=0$. (Requirement (i) is fulfilled.) Therefore $H^{(+)}$can be considered as the bosonic component and $H^{(-)}$as the fermionic. One can easily find that the $A\left(A^{+}\right)$ transforms the eigenfunction of $H^{(+)}\left(H^{(-)}\right)$into that of $H^{(-)}$ $\left(H^{(+)}\right)$, using Eqs. (11), (13) and (14),

$$
A \psi_{J K}^{(+)}(\varphi)=\sqrt{\mathrm{A}-\mathrm{B}} \hat{J}_{z} \hat{\Pi}_{+} \psi_{J K}^{(+)}(\varphi)=\sqrt{\mathrm{A}-\mathrm{B}} K \psi_{J K}^{(-)}(\varphi)
$$

and

$A^{+} \psi_{J K}^{(-)}(\varphi)=\sqrt{\mathrm{A}-\mathrm{B}} \hat{J}_{z} \hat{\Pi}_{-} \psi_{J K}^{(-)}(\varphi)$

$=\sqrt{\mathrm{A}-\mathrm{B}} K \psi_{J K}^{(+)}(\varphi)$

Note that $A \psi_{J K}^{(+)}(\varphi)=0$. (Requirement (iii) is fulfilled.)

The two partner Schrödinger equations are

$$
\begin{aligned}
& H^{(+)} \psi_{J K}^{(+)}(\varphi)=\left[(\mathrm{A}-\mathrm{B}) \hat{J}_{z}^{2} \hat{\Pi}_{+}\right] \psi_{J K}^{(+)}=E_{J K}^{(+)} \psi_{J K}^{(+)} \\
& K=0,1,2, \ldots
\end{aligned}
$$

and

$$
\begin{aligned}
& H^{(-)} \psi_{J K}^{(-)}(\varphi)=\left[(\mathrm{A}-\mathrm{B}) \hat{J}_{z}^{2} \hat{\Pi}_{-}\right] \psi_{J K}^{(-)}=E_{J K}^{(-)} \psi_{J K}^{(-)} \\
& K=1,2, \ldots
\end{aligned}
$$

where the rotational energy $E_{J K}^{(+)}=E_{J K}^{(-)}=\mathrm{B} J(J+1)+(\mathrm{A}-$ B) $K^{2}$. (Requirement (ii) is fulfilled.) Therefore the Hamiltonian $H_{S}$ is supersymmetric, and the bosonic $H^{(+)}$and the fermionic $H^{(-)}$are the partner Hamiltonians to each other. Since the difference between $H$ and $H_{S}$ is simply a constant term (see Eq. (8)), the Hamiltonian $H$ for a rigid symmetric top rotor has a supersymmetry.

\section{Conclusion and Discussions}

It is shown that the rotational motion of a rigid symmetric top molecule has a supersymmertric nature in it. For each $J$ rotational state there exists a supersymmetry spectrum, a non-degenerate zero energy ground state $(K=0)$ and pairs of excited states $(K$ and $-K)$. It is due to the time-reversal symmetry of rotation around the figure axis in the moleculefixed frame.

As mentioned earlier, we are not the first to observe that there exists a supersymmetry in a rotating body. Last year Rau suggested that there is a supersymmetric structure in the free rotor on a plane. The free rotor $\left(H=\hat{L}_{z}^{2} / 2 \mathrm{I}\right)$ discussed in Rau's work ${ }^{24}$ is mathematically equivalent to the symmetric top rotor $\left(H_{S}=(\mathrm{A}-\mathrm{B}) \hat{J}_{z}^{2}\right)$ dealt in this work. He used the time-reversal symmetry to construct the supercharges for the free rotor as we do the same for the symmetric top rotor. But we elaborate his idea in more detailed fashion to show the supersymmetry of a symmetric top rotor.

Rau also presented the supersymmetry of the free particle in one-dimension ${ }^{24}$ and he noted that the free particle system ( $\left.H=\left(-\hbar^{2} / 2 m\right)\left(\partial^{2} / \partial x^{2}\right)\right)$ is equivalent to the free rotor system $\left(H=\left(-\hbar^{2} / 2 \mathrm{I}\right)\left(\partial^{2} / \partial \varphi^{2}\right)\right)$. The only difference is that for the free particle the parity operator is used instead of the time-reversal operator. For the present symmetric top rotor case, the Eq. (12) introduces two eigenfunctions $\left(\psi_{J K}^{(+)}(\varphi)\right.$ and $\left.\psi_{J K}^{(-)}(\varphi)\right)$ of the time-reversal related projection operators $\hat{\Pi}_{+}$and $\hat{\Pi}_{-}$. Inserting Eq. (3) into Eq. (12), one obtains that

$$
\psi_{J K}^{(+)}(\varphi) \propto \cos (K \varphi) \text { and } \psi_{J K}^{(-)}(\varphi) \propto \sin (K \varphi) .
$$

One sees that $\psi_{J K}^{(+)}(\varphi)$ has an even parity and $\psi_{J K}^{(-)}(\varphi)$ has an odd parity. It is consistent with Eq. (13), which implies that one can use the parity operator in place of the timereversal operator for symmetric top rotor. Therefore the three systems, i.e., the current symmetric top rotor, the free particle in one-dimension, and the free rotor on a plane, are all equivalent. The supersymmetry of the free particle is explicitly shown in Ref. 27 where the general involution operator is introduced. For the free particle they used the parity operator as the involution operator while we use the time-reversal operator for the symmetric top rotor. Using the reflection operator as the involution operator, mathematically similar works were reported. ${ }^{28,29}$

The rotational structure of a rigid symmetric top rotor has been explicitly studied and is now a textbook subject. And the supersymmetry is also a well-established concept. Though this work does not suggest any new chemistry, the 
importance of this work lies in finding a supersymmetry in a non-trivial chemical system. (The supersymmetry related work was once published in this journal by the author. ${ }^{30}$ ) We believe that the search for supersymmetry in chemistry should be pursued in the future.

Acknowledgement. This work was supported by Korea Research Foundation Grant (KRF-2002-070-C00048).

\section{References}

1. Miller, D. Nature 1997, 385, 768.

2. Witten, E. Nature 2004, 429, 507.

3. Cooper, F.; Khare, A.; Sukhatme, U. P. Supersymmetry in Quantum Mechanics; World Scientific: Singapore, 2001.

4. Iachello, F. Phys. Rev. Lett. 1980, 44, 772.

5. Balantekin, A. B.; Bars, I.; Iachello, F. Phys. Rev. Lett. 1981, 47, 19.

6. Sourlas, N. Physica D 1985, 15, 115.

7. Cardy, J. L. Physica D 1985, 15, 123.

8. Tangerman, R. D.; Tjon, J. A. Phys. Rev. A 1993, 48, 1089.

9. Kostelecký, V. A.; Nieto, M. M. Phys. Rev. Lett. 1984, 53, 2285.

10. Rau, A. R. P. Phys. Rev. Lett. 1986, 56, 95.
11. Kostelecký, V. A.; Nieto, M. M. Phys. Rev. Lett. 1986, 56, 96.

12. Kostelecký, V. A.; Nieto, M. M. Phys. Rev. A 1985, 32, 1293.

13. Kostelecký, V. A.; Nieto, M. M. Phys. Rev. A 1985, 32, 3243.

14. Bluhm, R.; Kostelecký, V. A. Phys. Rev. A 1993, 47, 794.

15. Bluhm, R.; Kostelecký, V. A. Phys. Rev. A 1994, 49, 4628.

16. Sukumar, C. V. J. Phys. A: Math. Gen. 1985, 18, L697.

17. Jarvis, P. D.; Stedman, G. E. J. Phys. A: Math. Gen. 1986, 19, 1373.

18. Haymaker, R. W.; Rau, A. R. P. Am. J. Phys. 1986, 54, 928.

19. Lahiri, A.; Roy, P. K.; Bagchi, B. Int. J. Mod. Phys. 1990, 5, 1383.

20. Wigner, E. P. Group Theory; Academic Press: New York, 1959.

21. Tinkham, M. Group Theory and Quantum Mechanics; McGrawHill: New York, 1964.

22. Bunker, P. R. Molecular Symmetry and Spectroscopy; Academic Press: New York, 1979.

23. Zare, R. N. Angular Momentum; Wiley: New York, 1988

24. Rau, A. R. P. J. Phys. A: Math. Gen. 2004, 37, 10421.

25. Nikitin, A.; Champion, J. P. J. Mol. Spectrosc. 2005, 230, 168.

26. Cooper, F.; Kahre, A.; Sukhatme, U. P. Phys. Rep. 1995, 251, 267.

27. Cumbescure, M.; Gieres, F.; Kibler, M. J. Phys. A: Math. Gen. 2004, 37, 10385.

28. Plyushchay, M. S. Ann. Phys. 1996, 245, 339.

29. Plyushchay, M. S. Nucl. Phys. B 1999, 543, 447.

30. Sun, H. Bull. Korean Chem. Soc. 2005, 26, 1717. 